\title{
A NUMERICAL ALGORITHM BASED ON THE RCW METHOD TO SOLVE A SET OF FIRST-ORDER ORDINARY DIFFERENTIAL EQUATIONS
}

\author{
M. Rahmanzadeh', T. Asadi ${ }^{1}$, M. Atashafrooz ${ }^{2}$, \\ ${ }^{1}$ Department of Chemical Engineering, Sirjan University of Technology, Sirjan, Iran \\ ${ }^{2}$ Department of Mechanical Engineering, Sirjan University of Technology, Sirjan, Iran \\ e-mail: m.atashafrooz@sirjantech.ac.ir, Meysam.atashafrooz@yahoo.com \\ *corresponding author
}

\begin{abstract}
This research paper deals with a numerical algorithm based on the RCW method to solve a set of first-order ordinary differential equations. In this algorithm, the answer of each equation is considered as a polynomial of degree 2 . The coefficients used in this polynomial are categorized into groups of free and fixed coefficients. The free coefficients are obtained by optimizing the error function, while the fixed coefficients are computed from the magnitudes of derivative of this polynomial at initial value. To check the correctness of the current algorithm, its results for several case problems are compared with the findings of the Runge-Kutta (RK) method. This comparison shows that the algorithm presented in this paper has a higher accuracy and stability than the RK method even for the nonlinear problems.
\end{abstract}

Keywords: RCW method, ordinary differential equations, initial value problems, first-order equations

\section{Introduction}

Many physical phenomena, chemical reactions, mechanical systems, thermal systems and engineering applications can be simulated using the ordinary and partial differential equations (Zeeshan and Majeed 2016, Jaskulski et al. 2017, Atashafrooz et al. 2018, Reis et al. 2018, Sajjadi et al. 2018, Shah et al. 2018, Mahmoodabadi et al. 2018, Mehralian and Beni 2018, Kumar et al. 2018, Atashafrooz et al. 2019, Kang et al. 2019, Sajjadi et al. 2019, Rashid et al. 2019, Sheikholeslami et al. 2019, Atashafrooz and Asadi 2019).

In many cases, partial differential equations (PDEs) can be converted into a set of ordinary differential equations (ODEs) using spetial discretization (Esmaeilpour and Ganji 2007, Blinder 2013). Also, there are approaches to reduce the order of ordinary differential equations and to their change to a set of first-order ODEs (Ha 2001, Charroyer et al. 2018, Filipov et al. 2019). Therefore, finding an approach to solve a set of first-order ODEs is very important and practical.

So far, many scholars have focused on this topic and several different algorithms were presented to solve these equations, such as Euler's method, Taylor series algorithms, RungeKutta methods, improved Euler's method, multi-step approaches and the extrapolation method 
(Franco 2007, Brugnano and Magherini 2009, Cutolo et al. 2011, Tang and Sun 2012, Li and Wu 2016, Wang et al. 2017, Rahmanzadeh and Barfeie 2018, Korkmaz 2019, Amodio et al. 2019).

RCW method is an effective approach to solve an ordinary differential equation. This algorithm was firstly presented by Rahmanzadeh et al. (2013). Recently, RCW method was applied to simulate the Blasius problem (Rahmanzadeh et al. 2020). This problem simulates the viscous fluid flow over a semi-infinite flat plate and includes a nonlinear ordinary differential equation.

However, previous research has clearly shown that the RCW method has a high ability to solve the ODE problems. Therefore, the authors of the current research have decided to extend this method to solve a system of first-order initial-value problems. In other words, the main goal of this paper is to present a numerical algorithm based on the modified RCW method to solve a set of first-order ordinary differential equations. In the next sections, in addition to presenting the "Theory" and "Results and Discussions" parts, several case problems are considered and solved to ensure the correctness of the presented algorithm.

\section{Theory}

Consider a set of first-order differential equations as follows:

$$
\begin{aligned}
\frac{d y_{1}}{d t}=f_{1}\left(t, y_{1}, y_{2}, \cdots, y_{m}\right) & , y_{1}\left(t_{0}\right)=y_{01} \\
\frac{d y_{2}}{d t}=f_{2}\left(t, y_{1}, y_{2}, \cdots, y_{m}\right) & , y_{2}\left(t_{0}\right)=y_{02} \\
\vdots & \\
\frac{d y_{m}}{d t}=f 1\left(t, y_{1}, y_{2}, \cdots, y_{m}\right) & , y_{m}\left(t_{0}\right)=y_{0 m}
\end{aligned}
$$

To solve this system using RCW method, it is assumed that the solution of an equation in the above set is as follows:

$$
y_{i}=\frac{a_{i}}{2}\left(t-t_{0}\right)^{2}+a_{0 i}\left(t-t_{0}\right)+y_{01}, a_{0 i}=f_{i}\left(t_{0}, y_{01}, \cdots, y_{0 m}\right)
$$

Or

$$
y_{i}^{\prime}=a_{i}\left(t-t_{0}\right)+a_{0 i} \quad, i=1,2, \cdots, m
$$

Here, the $a_{i}$ variables $(i=1,2, \cdots, m)$ are named as the free coefficients. To calculate these coefficients, it is first necessary to define the residual functions $\left(R_{i}\right)$ as follows:

$$
\begin{gathered}
R_{1}=y_{1}^{\prime}-f_{1}\left(t, y_{1}, y_{2}, \cdots, y_{m}\right) \\
R_{2}=y_{2}^{\prime}-f_{2}\left(t, y_{1}, y_{2}, \cdots, y_{m}\right) \\
\vdots \\
R_{m}=y_{m}^{\prime}-f_{m}\left(t, y_{1}, y_{2}, \cdots, y_{m}\right)
\end{gathered}
$$

Then, an error function $\left(e_{i}\right)$ is defined based on these residual functions using the following equation: 


$$
e_{i}=\int_{t_{0}}^{t_{0}+h} R_{i}^{2} d t
$$

The next step to calculate the free coefficients is the optimization of this error function. This optimization is performed as follows:

$$
\begin{gathered}
d e_{1}\left(a_{1}, a_{2}, \cdots, a_{m}\right)=\int_{t_{0}}^{t_{0}+h} R_{1} \cdot \frac{\partial R_{1}}{\partial a_{1}} d t=0 \\
d e_{2}\left(a_{1}, a_{2}, \cdots, a_{m}\right)= \\
\int_{t_{0}}^{t_{0}+h} R_{2} \cdot \frac{\partial R_{2}}{\partial a_{2}} d t=0 \\
\vdots \\
d e_{m}\left(a_{1}, a_{2}, \cdots, a_{m}\right)=\int_{t_{0}}^{t_{0}+h} R_{m} \cdot \frac{\partial R_{m}}{\partial a_{m}} d t=0
\end{gathered}
$$

In fact, the values of $a_{1}, a_{2}, \cdots$ and $a_{m}$ are found by solving the above equations.

Besides, $a_{0 i}$ variables in Equations (2) and (3) are called fixed coefficients and based on the Equation set (1) their magnitudes are obtained from the values of $y_{i}^{\prime}$ at the $t_{0}$ point.

However, by replacing the fixed and free coefficients in Equation (2), the final answer of Equation system (1) can be computed as follows:

$$
y_{i}\left(t_{0}+h\right)=\int_{t_{0}}^{t_{0}+h} f_{i}\left(t, y_{1}, y_{2}, \cdots, y_{m}\right) \cdot d t \quad \mathrm{i}=1,2, \cdots, \mathrm{m}
$$

Accordingly, more details on the current algorithm needed to solve a set of first-order ordinary differential equations are presented as follows:

1- The matrix $F$ is defined and calculated as follows:

$$
F=\left[\begin{array}{llll}
F_{1} & F_{2} & \cdots & F_{m}
\end{array}\right]
$$

Where

$$
F_{i}=\left(t-t_{0}\right) \times\left(\frac{\left(t-t_{0}\right)}{2} \times \frac{\partial f_{i}}{\partial y_{i}}-1\right)
$$

2- The matrix $E$ is formed in the following manner:

$$
E=\left[\begin{array}{llll}
E_{1} & E_{2} & \cdots & E_{m}
\end{array}\right]=0
$$

Here

$$
E_{i}=R_{i} \cdot F_{i}=0
$$

In fact, the above equation leads to the formation of $m$ algebraic equations. Such that the free coefficients $\left(a_{i}\right)$ are obtained by solving these algebraic equations using the Newton method.

3- To calculate the values of $y_{i}\left(t_{0}+h\right)$, the following steps are carried out:

$$
t_{0 k}=t_{0}+(k-1) \frac{h}{4}, k=1, \cdots, 4
$$




$$
\begin{gathered}
y_{0 i 1}=y_{0 i} \\
a_{0 i k}=f_{i}\left(t_{0 k}, y_{01 k}, y_{02 k}, \cdots, y_{0 m k}\right) \\
y_{i}^{\prime}=a_{i}\left(t-t_{0 k}\right)+a_{0 i k} \\
y_{i}=\frac{a_{i}}{2}\left(t-t_{0 k}\right)^{2}+a_{0 i k}\left(t-t_{0 k}\right)+y_{0 i k} \\
J=\operatorname{Jacob}\left(\left[E_{1}, E_{2}, \cdots, E_{m}\right],\left[a_{1}, a_{2}, \cdots, a_{m}\right]\right)
\end{gathered}
$$

Here, $J=\operatorname{Jacob}\left(\left[E_{1}, E_{2}, \cdots, E_{m}\right],\left[a_{1}, a_{2}, \cdots, a_{m}\right]\right)$ is the Jacobin of $E_{i}$ against $a_{i}$ variables $(i=1,2, \cdots, m)$, which can be calculated as:

$$
J=\operatorname{Jacob}\left(\left[E_{1}, E_{2}, \cdots, E_{m}\right],\left[a_{1}, a_{2}, \cdots, a_{m}\right]\right)=\left[\begin{array}{ccc}
\frac{\partial E_{1}}{\partial a_{1}} & \cdots & \frac{\partial E_{1}}{\partial a_{m}} \\
\cdots & \ddots & \\
\frac{\partial E_{m}}{\partial a_{1}} & \cdots & \frac{\partial E_{m}}{\partial a_{m}}
\end{array}\right]
$$

By guessing the values of $a_{1}, a_{2}, \cdots, a_{m}$ and integrating of all arrays of matrices $E$ and $J$ relative to $t$ variable in the interval of $\left[t_{0} t_{0}+h\right]$, the new magnitudes of $a_{i}(i=1,2, \cdots, m)$ will be calculated as follows:

$$
\left[\begin{array}{c}
a_{1} \\
\vdots \\
a_{m}
\end{array}\right]=\left[\begin{array}{c}
a_{1} \\
\vdots \\
a_{m}
\end{array}\right]-\left[\int_{t_{0}}^{t_{0}+h} J d t\right]^{-1} \cdot \int_{t_{0}}^{t_{0}+h} E d t
$$

These calculations continue until the $a_{i}$ variables are converged. With this strategy, the values of $y_{0 i k+1}$ are obtained using the following equation:

$$
y_{0 i k+1}=\int_{t_{0 k}}^{t_{0 k}+h / 4} f_{i}\left(t, y_{1}, y_{2}, \cdots, y_{m}\right) \cdot d t+y_{0 i k}
$$

Finally, the value of $y_{0 i 5}$ is the values of $y_{i}\left(t_{0}+h\right)$.

\section{Results and Discussions}

In this section, the presented steps for solving a set of first-order ordinary differential equations using the RCW method are discussed in the form of a simple example.

Consider the following first-order ordinary differential equation:

$$
y_{1}^{\prime}=-20 y_{1}, y_{1}(0)=1
$$

First, function $F$ and matrix $E$ are calculated using the below equations:

$$
F=-10\left(t-t_{0}\right)^{2}-\left(t-t_{0}\right)
$$




$$
E=R_{1} \cdot\left[\left(t-t_{0}\right)+10\left(t-t_{0}\right)^{2}\right]
$$

Where

$$
R_{1}=20 y_{1}+y_{1}^{\prime}
$$

Then, value of $k$ set to one, such that the fixed coefficients are computed using the following equations:

$$
\begin{gathered}
t_{0 k}=t_{01}=t_{0}=0 \\
y_{01 k}=y_{011}=y_{01}=1 \\
a_{011}=f\left(t_{01}, y_{011}\right)=-20
\end{gathered}
$$

Therefore,

$$
\begin{gathered}
y_{1}^{\prime}=a_{1}\left(t-t_{01}\right)-a_{011}=a_{1} \cdot t-20 \\
y_{1}=\frac{a_{1}}{2} t^{2}-20 \cdot t+1
\end{gathered}
$$

In the next step, the magnitudes of $y$ and $y^{\prime}$ are replaced in matrix $E$, such that the values of this matrix are dependent on the $a_{1}$ and $t$ variables.

Then, matrix $J$ is formed in the following manner:

$$
J=j a c o b\left(E,\left[a_{1}\right]\right)=\left(10 t^{2}+t\right)^{2}
$$

Then, an arbitrary value is guessed for $a_{1}$ variable. With this strategy, matrixes $E$ and $J$ are dependent on the $t$ variable. By Integrating over $t$ variable in interval $\left[\begin{array}{ll}t_{0} & \mathrm{t}_{0}+h\end{array}\right]$, the new value of $a_{1}$ is numerically calculated as:

$$
a_{1}=a_{1}-\left[\int_{0}^{0.05} J \cdot d t\right]^{-1} \cdot \int_{0}^{0.05} E \cdot d t
$$

After the convergence of the numerical solution, the final value of $a_{1}$ is equal to. 289.4737. By substituting the $a_{1}$ in function $y_{1}$, the magnitude of $y_{012}$ is obtained as:

$$
y_{01 k+1}=y_{012}=\int_{0}^{0.05 / 4}-20 y_{1} \cdot d t+y_{011}=0.7794
$$

In next step, the value of $k$ set to 2 and the new coefficients are calculated as follows:

$$
\begin{gathered}
t_{02}=t_{0}+(k-1) \frac{h}{4}=\frac{0.05}{4} \\
y_{01 k}=y_{012}=0.7794 \\
a_{012}=f_{i}\left(t_{02}, y_{012}\right)=-15.5873 \\
y_{1}^{\prime}=a_{1}\left(t-t_{02}\right)-15.5873
\end{gathered}
$$




$$
y_{1}=a_{1}\left(t-t_{02}\right)-15.5873\left(t-t_{02}\right)+0.7794
$$

Then, $y_{013}$ can be calculated like the $k=1$ step:

$$
y_{013}=y_{012}+\int_{0.05 / 4}^{0.05 / 2} f_{1}\left(t, y_{1}\right) d t=0.6073
$$

Then, the calculations continue for $k=3$ and $k=4$.

According to what is done in $k=1$ to $k=4$ steps, the value of $y_{015}$ is obtained. In fact, the final value of $y_{1}$ is equal to $y_{015}$.

In the following, an attempt is made to show the influences of $k$ on the correctness of numerical solution.

The analytic solution of Equation (21) is:

$$
y_{\text {exact }}(t)=\exp (-20 t)
$$

Such that

$$
y_{\text {exact }}^{\prime}(t)=-20 \exp (-20 t)
$$

The values of $y_{\text {exac }}^{\prime}$ and $y_{1 k}^{\prime}(\mathrm{k}=1,2,3,4)$ are shown in Figure 1 . It should be mentioned that $y_{1 k}^{\prime}$ are estimated by a linear equation.

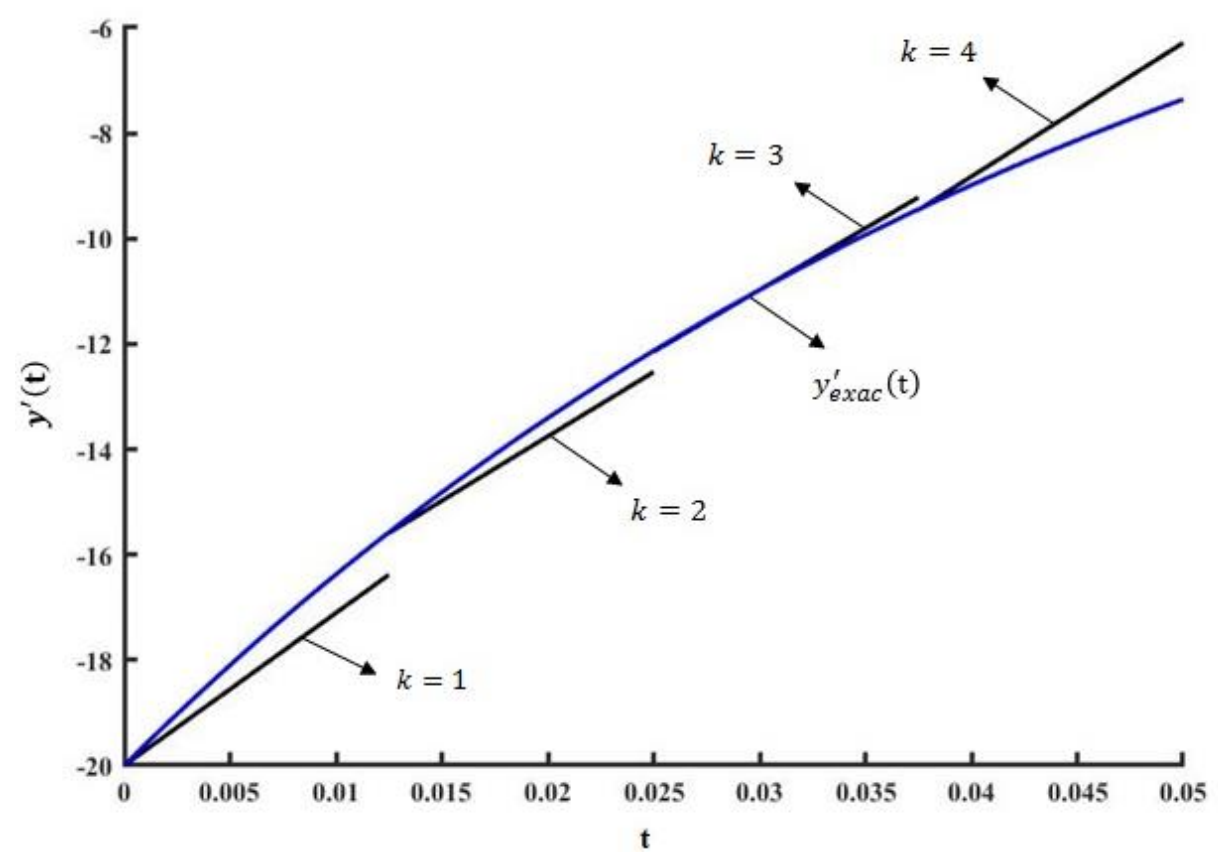

Fig. 1. The values of $y_{\text {exac }}^{\prime}$ and $y_{1 k}^{\prime}(\mathrm{k}=1,2,3,4)$

As it is shown in Figure 1, the values of $y_{11}^{\prime}$ and $y_{12}^{\prime}$ are lower than $y_{\text {exac }}^{\prime}(t)$, whilst $y_{13}^{\prime}$ and $y_{14}^{\prime}$ have higher values than $y_{\text {exac }}^{\prime}(t)$. 
Given that error values between the exact solution $\left(y_{\text {exac }}^{\prime}\right)$ and the estimated solutions $\left(y_{1 k}^{\prime}\right)$ can be positive and negative, it can be concluded that the values of final error at the desired point $\left(t_{0}+h\right)$ is decreased by summing the positive and negative errors.

However, it can be seen from Figure 2 that the absolute magnitude of error is very low for $k=4$.

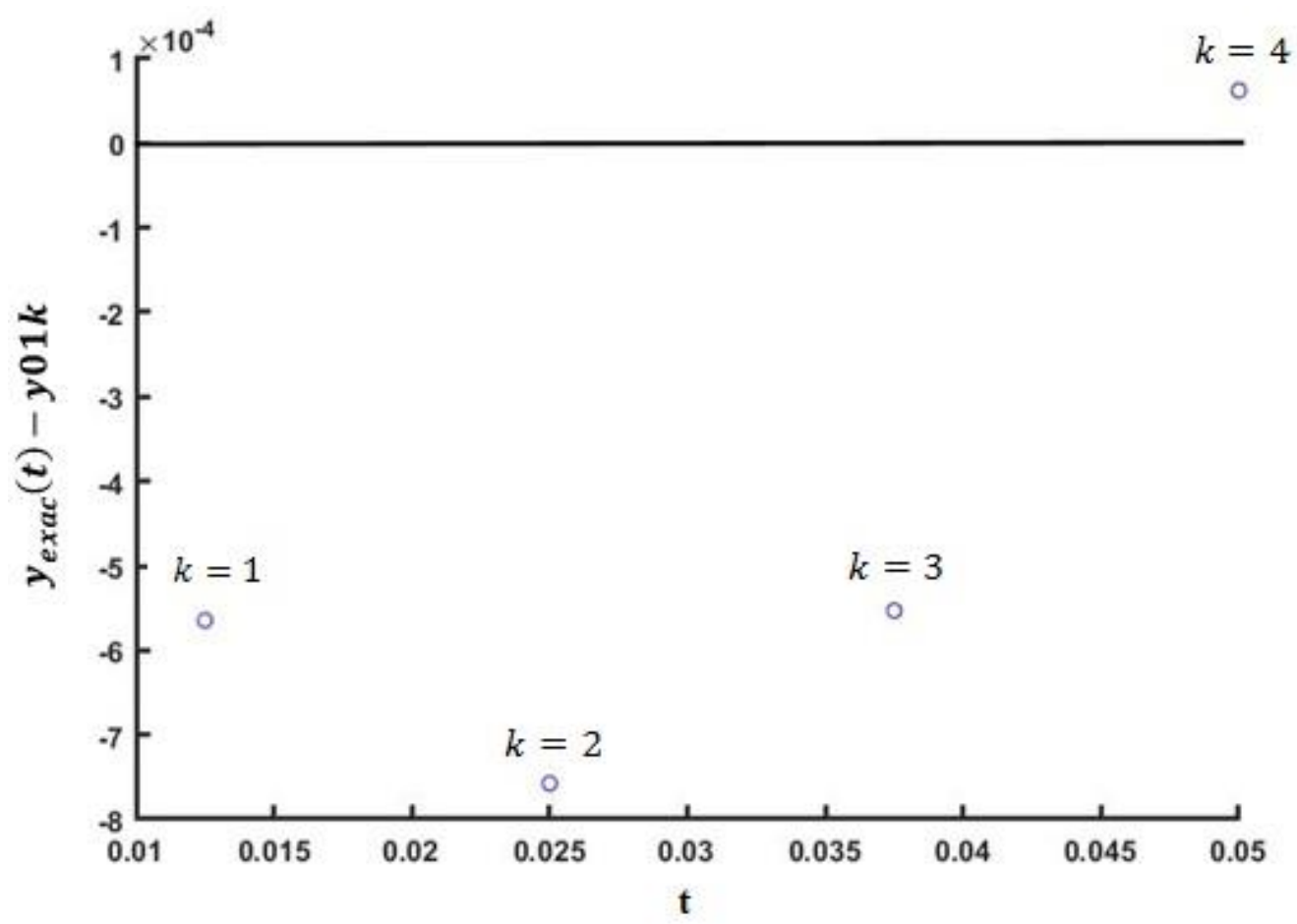

Fig. 2. The error magnitudes for different values of $k$

\section{Examples}

In this section, three different problems are selected to ensure the accuracy of the present approach for solving a set of first-order ordinary differential equations. In all the problems, the equations system is solved using the RCW and Runge-Kutta (RK) methods. In fact, these solutions are performed to compare the error values of these two methods.

\subsection{Problem I}

Consider the following equations system:

$$
\begin{array}{ll}
y_{1}^{\prime}=9 y_{1}+24 y_{2}+5 \cos (t)-\frac{1}{3} \sin (t), & \mathrm{y}_{1}(0)=\frac{4}{3} \\
y_{2}^{\prime}=-24 y_{1}-51 y_{2}-9 \cos (t)+\frac{1}{3} \sin (t), & \mathrm{y}_{2}(0)=\frac{2}{3}
\end{array}
$$


The errors magnitudes of RCW and RK methods in computing $y_{1}$ and $y_{2}$ variables are presented in Table 1. These results are obtained for the case of $h=0.02$. As it can be seen from this table, the RCW method is more accurate than the RK method.

\begin{tabular}{|c|c|c|c|c|}
\hline \multirow{2}{*}{$\mathrm{t}$} & \multicolumn{2}{|c|}{ RCW method } & \multicolumn{2}{c|}{ RK method } \\
\cline { 2 - 5 } & Error of $y_{1}$ & Error of $y_{2}$ & Error of $y_{1}$ & Error of $y_{2}$ \\
\hline 0.02 & $2.54396 \times 10^{-5}$ & $-6.61495 \times 10^{-5}$ & 0.002125136 & -0.00425029 \\
\hline 0.04 & $1.83852 \times 10^{-5}$ & $-5.81536 \times 10^{-5}$ & 0.001952972 & -0.003905981 \\
\hline 0.06 & $6.85835 \times 10^{-6}$ & $-3.70662 \times 10^{-5}$ & 0.001346125 & -0.002692301 \\
\hline 0.08 & $-1.95706 \times 10^{-6}$ & $-1.95492 \times 10^{-5}$ & 0.000824814 & -0.001649691 \\
\hline 0.1 & $-7.36233 \times 10^{-6}$ & $-8.04911 \times 10^{-6}$ & 0.000473872 & -0.000947818 \\
\hline
\end{tabular}

Table 1. The errors magnitudes of RCW and RK methods in computing $y_{1}$ and $y_{2}$ variables for Problem I

\subsection{Problem II}

Consider a set of nonlinear ordinary differential equations as below:

$$
\begin{array}{ll}
\frac{d y_{1}}{d t}=\frac{3 y_{1} y_{2}}{t^{3}}+y_{1}^{2}-e^{6 t}, & \mathrm{y}_{1}(1)=\mathrm{e}^{3} \\
\frac{d y_{2}}{d t}=3 y_{2}^{\frac{2}{3}}+\log \left(y_{1}\right)-3 t, & \mathrm{y}_{2}(1)=1
\end{array}
$$

Where the exact solutions are $y_{1, \text { exact }}(t)=e^{3 t}$ and $y_{2, \text { exact }}(t)=t^{3}$.

The amounts of $y_{1}$ and $y_{2}$ obtained from the RCW and RK methods are shown in Figure 3 for the case of $h=0.02$. These findings are compared with the results of exact solutions.

The detailed analysis of this figure clearly shows that the accuracy and stability of the RCW method is higher than the RK method. In fact, the RK method loses its stability at the point of $t=1.16$. 

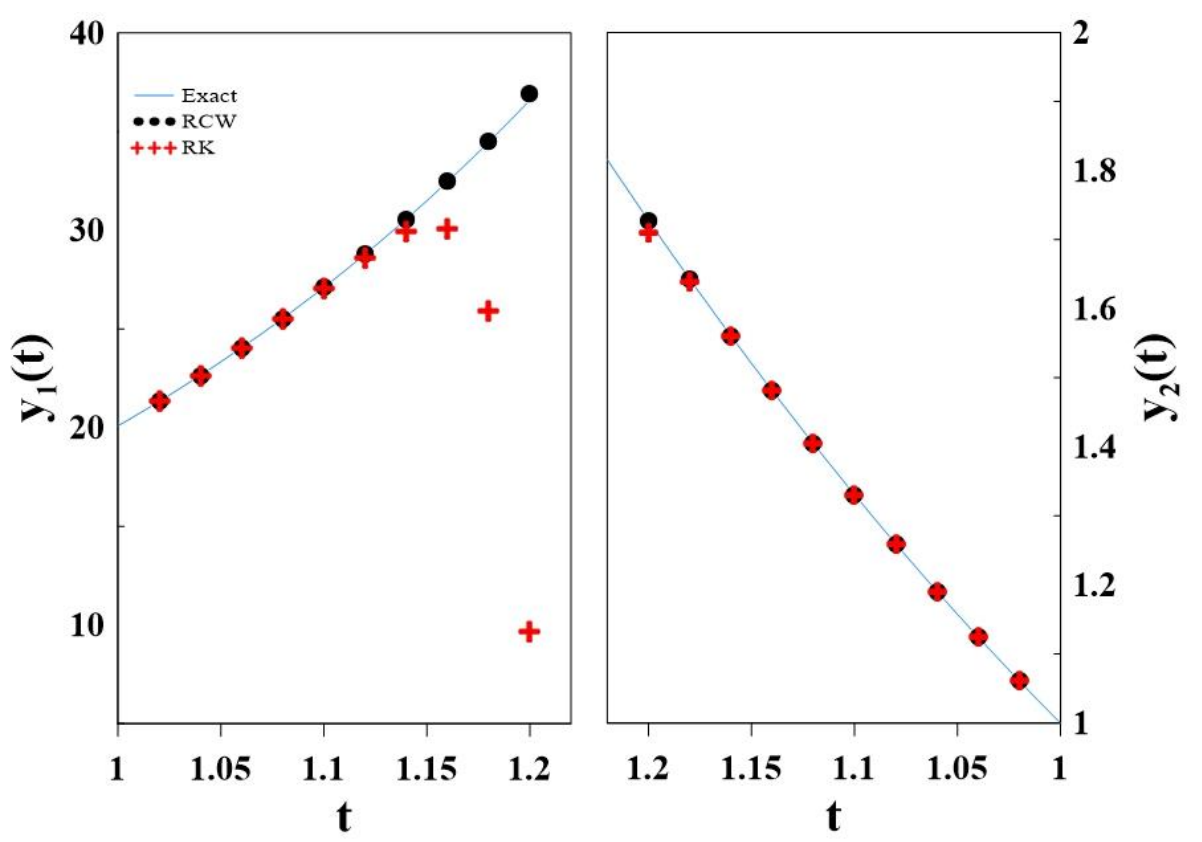

Fig. 3. Comparison of $y_{1}$ and $y_{2}$ amounts obtained from the RCW and RK methods with the results of the exact solutions for Problem II

\subsection{Problem III}

Consider a set of nonlinear, time dependent ordinary differential equations as follows:

$$
\begin{array}{ll}
\frac{d y_{A}}{d t}=-k_{1} y_{A} y_{B}, & y_{A 0}=55 \\
\cdot \cdot & \\
\frac{\mathrm{dy}_{\mathrm{R}}}{d t}=k_{1} y_{A} y_{B}-k_{2} y_{B} y_{R}, & y_{R 0}=0 \\
\frac{\mathrm{dy}_{\mathrm{s}}}{d t}=k_{2} y_{B} y_{R}, & y_{s 0}=0
\end{array}
$$

Here, $y_{A}, y_{B}, y_{R}$ and $y_{s}$ variables point to the concentration of various materials, while $y_{A 0}, y_{B 0}, y_{R 0}$ and $y_{s 0}$ parameters are initial concentration of these materials. In fact, this set of equations is related to chemical reactions in a batch reactor.

The values of relative error for $y_{A}$ variable in both RCW and RK methods are presented in Table 2. These values are tabulated for different values of $h$. As it is seen from this table, the accuracy of the RK method decreases considerably by increasing the amounts of $h$. In fact, Table 2 clearly shows that the stability of the RK method is not suitable at high values of $h$. However, the accuracy and stability of the RCW method for all presented step lengths is very good.

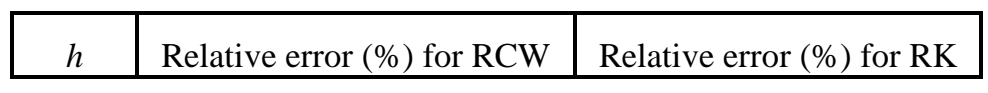




\begin{tabular}{|l|l|l|}
0.006 & 0.191091 & 1.848575 \\
\hline 0.007 & 0.33683 & 6.235279 \\
\hline 0.008 & 0.555213 & 17.00306 \\
\hline 0.009 & 0.876214 & 40.20436 \\
\hline 0.01 & 1.346192 & 85.41022 \\
\hline
\end{tabular}

Table 2. Values of relative error for $y_{A}$ variable in both RCW and RK methods for Problem III

\section{Conclusion}

In this research, an attempt is made to present a numerical approach based on the RCW method to solve a set of first-order ordinary differential equations. To reach this goal, a polynomial of degree 2 is considered as the answer of each equation. This polynomial includes two groups of free and fixed coefficients. The fixed coefficients are obtained from the derivative magnitudes of this polynomial at initial values, while the free coefficients are computed by optimizing the error function. To provide more details about the mentioned algorithm, all the steps of the numerical solution are described in the form of a sample example. Besides, the findings of the current algorithm for three case problems are compared with the results of the Runge-Kutta method. This comparison clearly shows that the approach used in this paper has a higher stability and accuracy than the Runge-Kutta method even for the nonlinear problems. In addition, the results of the RCW method are in an excellent agreement with the findings of the exact solution.

\section{References}

Amodio P, Brugnano L and Iavernaro F (2019). Analysis of spectral Hamiltonian boundary value methods (SHBVMs) for the numerical solution of ODE problems, Numerical Algorithms, First online, 1-20 (https://doi.org/10.1007/s11075-019-00733-7).

Atashafrooz M, Bahaadini R and Sheibani HR (2018). Nonlocal, strain gradient and surface effects on vibration and instability of nanotubes conveying nanoflow, Mechanics of Advanced Materials and Structures, First online, 1-13 (https://doi.org/10.1080/15376494.2018.1487611).

Atashafrooz M and Asadi T (2019). The effects of buoyancy force on the irreversibility of threedimensional step flow in an inclined duct, Journal of the Serbian Society for Computational Mechanics, 13(1), 1-16.

Atashafrooz M, Sheikholeslami M, Sajjadi H and Delouei AA (2019). Interaction effects of an inclined magnetic field and nanofluid on forced convection heat transfer and flow irreversibility in a duct with an abrupt contraction, Journal of Magnetism and Magnetic Materials, 478, 216-226.

Blinder SM (2013). Guide to essential math: a review for physics, chemistry and engineering students. Newnes.

Brugnano L and Magherini C (2009). Recent advances in linear analysis of convergence for splittings for solving ODE problems, Applied Numerical Mathematics, 59, 542-557.

Charroyer L, Chiello O and Sinou JJ (2018). Self-excited vibrations of a non-smooth contact dynamical system with planar friction based on the shooting method, International Journal of Mechanical Sciences, 144, 90-101. 
Cutolo A, Piccoli B and Rarità L (2011). An upwind-Euler scheme for an ODE-PDE model of supply chains, SIAM Journal on Scientific Computing, 33(4), 1669-1688.

Esmaeilpour M and Ganji DD (2007). Application of He's homotopy perturbation method to boundary layer flow and convection heat transfer over a flat plate, Physics Letters A, 372(1), 33-38.

Filipov SM, Gospodinov ID and Faragó I (2019). Replacing the finite difference methods for nonlinear two-point boundary value problems by successive application of the linear shooting method, Journal of Computational and Applied Mathematics, 358, 46-60.

Franco JM (2007). Exponentially fitted symplectic integrators of RKN type for solving oscillatory problems, Computer Physics Communications, 177(6), 479-492.

Ha SN (2001). A nonlinear shooting method for two-point boundary value problems, Computers \& Mathematics with Applications, 42(10-11), 1411-1420.

Jaskulski M, Atuonwu JC, Tran TTH, Stapley AGF and Tsotsas E (2017). Predictive CFD modeling of whey protein denaturation in skim milk spray drying powder production, Advanced Powder Technology, 28(12), 3140-3147.

Kang J, Zhang D, Zhou F, Li H and Xia T (2019). Numerical modeling and experimental validation of fractional heat transfer induced by gas adsorption in heterogeneous coal matrix, International Journal of Heat and Mass Transfer, 128, 492-503.

Korkmaz A (2019). Explicit exact solutions to some one-dimensional conformable time fractional equations, Waves in Random and Complex Media, 29(1), 124-137.

Kumar MS, Sandeep N, Kumar BR and Saleem S (2018). Effect of aligned magnetic field on MHD squeezing flow of Casson fluid between parallel plates, In Defect and Diffusion Forum 384, 1-11.

Li YW and Wu X (2016). Functionally fitted energy-preserving methods for solving oscillatory nonlinear Hamiltonian systems, SIAM Journal on Numerical Analysis, 54(4), 2036-2059.

Mahmoodabadi MJ, Mahmoodabadi F and Atashafrooz M (2018). Development of the meshless local Petrov-Galerkin method to analyze three-dimensional transient incompressible laminar fluid flow, Journal of the Serbian Society for Computational Mechanics, 12(2), 128-152.

Mehralian F. and Beni YT (2018). Vibration analysis of size-dependent bimorph functionally graded piezoelectric cylindrical shell based on nonlocal strain gradient theory, Journal of the Brazilian Society of Mechanical Sciences and Engineering, 40(1), 27.

Rahmanzadeh M, Cai L and White RE (2013). A new method for solving initial value problems, Computers \& Chemical Engineering, 58, 33-39.

Rahmanzadeh M and Barfeie M (2018). An explicit time-stepping method based on error minimization for solving stiff system of ordinary differential equations, Malaysian Journal of Mathematical Sciences, 12(2), 267-283.

Rahmanzadeh M, Asadi T and Atashafrooz M (2020). The development and application of the RCW method for the solution of the Blasius problem, Journal of Applied and Computational Mechanics, 6(1), 105-111.

Rashid M, Khan MI, Hayat T, Khan MI and Alsaedi A, (2019). Entropy generation in flow of ferromagnetic liquid with nonlinear radiation and slip condition, Journal of Molecular Liquids, 276, 441-452.

Reis M, Kromer JA and Klipp E (2018). General solution of the chemical master equation and modality of marginal distributions for hierarchic first-order reaction networks, Journal of mathematical biology, 77(2), 377-419.

Sajjadi H, Delouei AA, Atashafrooz M and Sheikholeslami M (2018). Double MRT Lattice Boltzmann simulation of 3-D MHD natural convection in a cubic cavity with sinusoidal temperature distribution utilizing nanofluid, International Journal of Heat and Mass Transfer, 126, 489-503. 
Sajjadi H, Delouei AA, Sheikholeslami M, Atashafrooz M and Succi S (2019). Simulation of three dimensional MHD natural convection using double MRT Lattice Boltzmann method, Physica A: Statistical Mechanics and its Applications, 515, 474-496.

Shah Z, Gul T, Islam S, Khan MA, Bonyah E, Hussain F, Mukhtar S and Ullah M (2018). Three dimensional third grade nanofluid flow in a rotating system between parallel plates with Brownian motion and thermophoresis effects, Results in Physics, 10, 36-45.

Sheikholeslami M, Sajjadi H, Delouei AA, Atashafrooz M and Li Z (2019). Magnetic force and radiation influences on nanofluid transportation through a permeable media considering $\mathrm{Al}_{2} \mathrm{O}_{3}$ nanoparticles, Journal of Thermal Analysis and Calorimetry, 136(6), 2477-2485.

Tang W and Sun Y (2012). Time finite element methods: A unified framework for numerical discretizations of ODEs, Applied Mathematics and Computation, 219(4), 2158-2179.

Wang B, Meng F and Fang Y (2017). Efficient implementation of RKN-type Fourier collocation methods for second-order differential equations, Applied Numerical Mathematics, 119, 164178.

Zeeshan A and Majeed A (2016). Heat transfer analysis of jeffery fluid flow over a stretching sheet with suction/injection and magnetic dipole effect, Alexandria Engineering Journal, 55, 2171-2181. 